\title{
Self-care management strategies among individuals living with type 2 diabetes mellitus: nursing interventions
}

This article was published in the following Dove Press journal:

Nursing: Research and Reviews

26 July 2013

Number of times this article has been viewed

\section{Caralise W Hunt}

Auburn University School of Nursing, Auburn, AL, USA
Correspondence: Caralise W Hunt Auburn University School of Nursing, 219 Miller Hall, Auburn 36849, AL, USA

Tel +l 3348446763

Fax + I 3348445654

Email huntcar@auburn.edu
Abstract: Nurses provide care for individuals living with diabetes in a variety of areas. Nursing interventions assist individuals living with diabetes to manage diabetes and can positively affect outcomes. This article describes an integrated literature review conducted to evaluate and summarize nursing interventions and research in self-management of type 2 diabetes mellitus. PubMed, PsycINFO, CINAHL, and the Cochrane database were searched for the years 2002-2013 using the search terms "diabetes", "type 2 diabetes", "self-care", "self-management", "diabetes selfmanagement", "intervention", and "nursing". Results from the review indicate that nurses deliver care independently and in conjunction with other health care providers for individuals living with diabetes. A majority of the reviewed studies included a nursing education intervention for patients living with diabetes. Nursing interventions are linked to improvements in diabetes knowledge, self-management behaviors, and physiologic and psychologic outcomes.

Keywords: type 2 diabetes mellitus, self-care management, nursing interventions

\section{Background}

Effective diabetes management necessitates involvement of a collaborative and integrated team working with the patient to establish goals and implement individualized care. The American Diabetes Association recommends that individuals with diabetes receive coordinated care from a team that includes doctors, nurse practitioners, physician's assistants, nurses, dieticians, pharmacists, and mental health professionals. ${ }^{1}$ Nurses are integral members of this interdisciplinary team and often serve as the primary care managers. The literature describes a variety of areas in which nurses care for patients living with diabetes, including providing psychologic support, assisting with psychomotor skills, teaching diabetes care, promoting self-management, and setting goals and priorities for patient-centered, individualized care. ${ }^{2}$ Nursing interventions assist individuals living with diabetes to understand and manage diabetes and can positively affect outcomes. ${ }^{3}$ Nurses view individuals living with diabetes holistically and consider the physiologic, psychologic, social, and environmental components of diabetes that are needed to achieve optimal outcomes. This integrated literature review was conducted to evaluate and summarize nursing interventions and research in self-management of type 2 diabetes mellitus.

\section{Search methods}

PubMed, PsycINFO, CINAHL, and the Cochrane database were searched for the years 2002-2013. The search was limited to articles written in English and included adult participants with type 2 diabetes mellitus. Keywords included “diabetes”, "type 2 diabetes", 
"self-care", "self-management", "diabetes self-management", "intervention", and "nursing". The online search was supplemented by a review of references identified from retrieved articles. Three hundred and sixteen results were identified from the searches. Duplicate citations were eliminated. Studies that did not include nurses in development or implementation of the intervention were excluded. Articles describing a study without reporting the findings were also eliminated. A total of 40 publications were reviewed, including 29 randomized controlled trials, six quasiexperimental designs, and five nonexperimental pretest and post-test design studies. A table summarizing study purpose, description of the intervention and nurses' role, and major study results is available. http://www.dovepress.com/ cr data/supplementary file 49406.pdf.

\section{Role of nurses in diabetes self-management interventions}

Nurses are in a position to champion transformation of chronic care, including diabetes management. ${ }^{4}$ Nursing interventions are aimed at prevention of chronic conditions as well as treatment of current chronic conditions..$^{5}$ Nurses play an important role in ensuring success of diabetes interventions by supervising delivery of care, directly delivering care, and coaching nonmedical personnel in supporting diabetes care for a variety of patient populations. Studies in which nurses supervise delivery of care include various approaches and are developed and implemented by nurses. Nurses also directly deliver care as part of an interprofessional team. Many of these interventions include an educational component. Coaching nonmedical personnel involves supporting community health advisors as they work with patients.

\section{Supervising delivery of care}

A variety of approaches are developed and implemented by nurses in diverse settings. Sherifall et al developed and implemented a study to evaluate the effects of a computergenerated, patient-tailored feedback intervention on glycemic control. ${ }^{6}$ Another nurse-led and implemented study designed to improve glycemic control examined the effect of a hospital-based clinic intervention. ${ }^{7}$ Kang et al compared the effectiveness of a family partnership intervention with conventional care for managing diabetes. ${ }^{8}$ A nurse-led and implemented study focused on enhancing self-efficacy, a factor that is significantly linked to improving diabetes self-management. ${ }^{9}$ Nurse-led and implemented studies also focus on culturally appropriate diabetes interventions. ${ }^{10-14}$

Several studies developed and implemented by nurses have involved an educational intervention. New conducted focus groups for people living with type 2 diabetes mellitus to identify educational needs and developed an educational intervention based on focus group findings. ${ }^{15}$ Skelly et al developed a symptom-based educational intervention that addressed diabetes symptoms and allowed participants to select self-management strategies for symptoms based on unique needs and preferences. ${ }^{16}$ Song et al developed a web-based diabetes self-management education program that allowed participants to proceed through modules at their own pace and remediate desired aspects. ${ }^{17}$

Educational intervention studies are designed to evaluate the effects of specific diabetes education programs on outcomes including diabetes knowledge, self-management activities, and glycosylated hemoglobin $\left(\mathrm{HbA}_{1 \mathrm{c}}\right) \cdot{ }^{15,18,19}$ Other studies evaluate effects of novel educational approaches including tailored educational programs, web-based diabetes education, multimedia educational programs, and telephonedelivered education. ${ }^{13,16,17,20,21}$

\section{Delivering care}

As members of an interprofessional health care team, nurses implement a variety of strategies to assist patients with diabetes self-management. Nurses and nutritionists frequently work together to assist patients with appropriate food choices and intake which is one of the most difficult areas of diabetes self-management. ${ }^{5,15,22,23}$ Nurses also work in conjunction with psychologists to provide counseling for patients living with diabetes. ${ }^{24}$ Nurses work closely with physicians to monitor patients and make alterations in the plan of care as needed. ${ }^{18,25}$

The American Diabetes Association recommends that individuals with diabetes should receive diabetes self-management education according to National Standards for Diabetes Self-Management Education and Support at diagnosis and as needed thereafter. ${ }^{1}$ Diabetes management education is provided by nurses using a wide range of methods. Both individual and group sessions are conducted. 3,5,7,10-12,14,19,22,24-27 Education may also be delivered using a web-based format. ${ }^{6,17,28}$ Education for family members or significant others of those living with diabetes is recommended and is included in nursing education. ${ }^{14,29}$

Diabetes education and support is frequently provided through telephone counseling sessions. ${ }^{30-32}$ Participants in one study were contacted at least once a week for a 12-week period by a registered nurse. The calls consisted of discussion of diet, exercise, medications, and self-monitoring of blood glucose. Participants were allowed to ask questions and adjustments to the treatment plan were made as needed based 
on the blood glucose log and dietary intake. ${ }^{23}$ Another study utilized weekly, prerecorded telephone calls to participants. An automated health education message or nurse phone follow-up occurred based on participant responses during these calls. ${ }^{33}$ Telephone counseling provides individualized care for people living with diabetes as demonstrated in a study in which participants completed questionnaires related to diabetes self-management. Participant answers to these questions were used to guide telephone sessions and provide individualized support and instruction. ${ }^{18}$

Nurses can provide education and monitoring through telephone interventions. In a study designed to offer support for diabetes self-management, a telehealth nurse monitored blood glucose values and insulin doses using the web. Patient data were reviewed and the telehealth nurse telephoned participants as needed based on the data to recommend changes in the treatment plan or follow-up with the primary health care provider. ${ }^{34}$

Tailored interventions involve customizing interventions to meet individual needs, abilities, and preferences, and require nurses to assist patients to follow a recommended treatment plan. ${ }^{35}$ Nurses deliver tailored educational plans based on assessment and patient-developed goals for diabetes management. ${ }^{8,18,29}$ Educational sessions may be guided by participants' diabetes self-management questions and experiences. Nurses emphasize experiential learning and work with participants to set goals and problem-solve for self-management. ${ }^{24,36}$

Nurses serve as coaches for patients living with diabetes as they navigate through the self-management treatment plan. As coaches, nurses assist patients to identify personal goals for self-management. ${ }^{37}$ The coaching process is ongoing. As patients encounter problems or questions, nurses can help patients adjust goals or problem-solve using their own diabetes knowledge or with the assistance of algorithms developed by the health care team. ${ }^{25,38}$

\section{Coaching nonmedical personnel}

Community health advisors, or lay health workers, can facilitate diabetes education and care. ${ }^{39}$ Nurses provide education and support for community health advisors. ${ }^{11,14}$ In a study to test the feasibility of using promotoras to deliver a diabetes self-management intervention, nurses spent 8 weeks educating and training promotoras. Following completion of training, the promotora and nurse delivered a diabetes educational course. The course was followed by home visits from promotoras to present tailored educational sessions with the nurse available for consultation as needed. ${ }^{12}$
Telecarers can be educated and trained to provide diabetes self-management support. Nurses educate and supervise telecarers who provide calls to participants regarding diabetes knowledge, readiness to change behaviors, medication adherence, and blood glucose control.$^{40} \mathrm{~A}$ similar approach was used in a study to compare telephone and print diabetes selfmanagement interventions. Health educators were trained and supervised by a diabetes nurse educator. Health educator calls focused primarily on diabetes medication adherence, but also included lifestyle changes through healthy eating and physical activity. ${ }^{21}$

\section{Effects of nurse intervention on patient outcomes}

The effect of nursing interventions is measured using a variety of outcomes. In addition to physiologic measures assessing clinical management of diabetes, other outcome measures evaluated in nursing intervention studies include diabetes knowledge, self-management behaviors, psychosocial factors, and psychologic variables.

\section{Diabetes knowledge}

To evaluate the effect of education, diabetes knowledge is often measured in nursing intervention studies. A nursedeveloped and implemented study that included 52 contact hours over 12 months of support and instructional sessions on diabetes basics and self-management found significant increases in diabetes knowledge compared with a wait-listed control group. ${ }^{10}$ Another study evaluated the effects of interactive multimedia on self-directed learning in patient's knowledge of diabetes using a collection of medical and nursing instructions about diabetes recorded on compact discs. The control group received a diabetes self-management booklet and counseling. Significant improvements were noted for diabetes knowledge in the intervention group, but not in the control group..$^{20} \mathrm{An}$ intervention that emphasized family participation provided individual and group educational sessions and monthly telephone discussions, while the control group had usual care. Knowledge scores for the intervention group were significantly higher than for the control group. ${ }^{8}$ In a study evaluating the efficacy of ongoing group-based diabetes selfmanagement education, a nurse provided educational sessions focused on type 2 diabetes basics, complications, diet, physical activity, and improving metabolic control. Significantly higher levels of diabetes knowledge were noted in the intervention group. ${ }^{27}$ Several other studies found significant improvements in diabetes knowledge for the experimental group following educational interventions. ${ }^{14,17-19,31}$ 
Interventions that provide diabetes education in differing formats for experimental and control groups often note improvements in both groups. One study provided a video behavior support intervention with workbook and telephone coaching sessions by a registered nurse for the experimental group and a brochure on diabetes self-management for the control group. Both groups showed significant increases with no difference between groups. ${ }^{37} \mathrm{~A}$ study that provided patient-centered, needs-driven educational and informational sessions for the experimental group and typical diabetes selfmanagement education for the control group noted slight increases in diabetes knowledge, but no difference between groups..$^{15}$ A pilot study tested the feasibility of a cardiac and diabetes self-management program that provided three educational sessions followed by a telephone call from a nurse one week after discharge and text-messaging one week after telephone contact to address questions related to selfmanagement. This intervention noted slight improvements in diabetes knowledge, but no difference between experimental and control groups. ${ }^{41}$

\section{Self-management behaviors}

People living with diabetes must assume an active role in the planning and implementation of their care. ${ }^{1}$ Self-management requires appropriate support for patients from health care professionals to enable them to manage diabetes confidently and competently. ${ }^{42}$ Nurses can facilitate and assist patients to set goals and problem-solve for management of diabetes. ${ }^{43}$ The American Diabetes Association recommends that individuals living with diabetes receive support for selfmanagement behaviors including healthy eating, physical activity, self-monitoring of blood glucose, medication-taking, and monitoring for complications. Many nursing interventions implement approaches that support patient behavior change efforts. ${ }^{1}$

One study describes implementation of a nurse-directed intervention to promote healthy behaviors in adults with diabetes. The patient education program included content presentation, discussion, problem-solving exercises, and visual aids. Statistically significant improvements in the health promoting behaviors of diet, exercise, medicationtaking, hygiene, and prevention of complications were noted for the experimental group when compared with the usual care control group. ${ }^{3}$ An intervention that was cocreated with adults living with type 2 diabetes noted significant improvements in the experimental group for diet, exercise, medication adherence, and glucose self-monitoring. ${ }^{15}$ An in-home, nurse-delivered, symptom-focused teaching and counseling intervention provided diabetes management intervention modules for people living with type 2 diabetes. Significant improvements in medication, diet, and glucose monitoring practices were noted for the intervention group. There was no statistically significant difference between groups for exercise ${ }^{16}$ Another study assessed the effectiveness of a structured diabetes education program on self-care. Face-toface and telephone educational sessions provided by nurses addressed self-care and problem-solving. Self-monitoring of blood glucose and participation in physical activity significantly improved in the intervention group and the change was also significant when compared with the control group. ${ }^{8}$ In a 24-month study, a certified diabetes education nurse and psychologist cofacilitated sessions that focused on diabetes self-management questions, experiential learning, coping, goal-setting, and problem-solving. After 6 months, significant improvements were noted for diet, monitoring blood glucose, and inspecting feet for complications. After 24 months, significant improvements were found for diet and insulin use. ${ }^{24}$ Nurse intervention studies often find significant improvements for self-management behaviors in both intervention and control groups. ${ }^{6,13,17,18,37,44}$

\section{Physiologic outcomes}

Physiologic outcomes of nurse intervention studies include $\mathrm{HbA}_{1 \mathrm{c}}$, fasting blood glucose, lipids, body mass index (BMI), weight, and blood pressure. Following a nurse-developed and implemented instructional and support intervention, $\mathrm{HbA}_{1 \mathrm{c}}$, fasting blood glucose, lipids, and BMI improved significantly. ${ }^{10} \mathrm{~A}$ telehealth intervention with nurse-led educational sessions found significant improvements in $\mathrm{HbA}_{1 \mathrm{c}}$ and low-density lipoproteins in the experimental group. No difference was noted for blood pressure or BMI. ${ }^{22}$ A study that explored the effectiveness of an advanced practice nurse-led follow-up telephone intervention on glycemic control found significant improvements in daily fasting blood glucose results compared with the control group. ${ }^{25}$ Another study designed to test the efficacy of a controlled nursing intervention centered on education and counseling noted significant improvement in $\mathrm{HbA}_{1 \mathrm{c}}$ in the intervention group compared with the control group. ${ }^{26}$ Researchers examined whether a multifaceted, culturally sensitive, primary carebased behavioral intervention implemented by a nurse case manager and/or a community health advisor could improve $\mathrm{HbA}_{1 \mathrm{c}}$, lipids, and blood pressure. The combined nurse and community health advisor team was most effective, with significant decreases noted in diastolic blood pressure and lipids. A decrease in $\mathrm{HbA}_{1 \mathrm{c}}$ occurred, but not to a level 
of significance. ${ }^{11}$ In a study designed to reduce cardiovascular risk in patients with type 2 diabetes, a nurse led a course that included both individual and group sessions to discuss self-care domains and assist participants to establish and meet self-care goals. BMI and systolic blood pressure were significantly decreased in the intervention group in this study. ${ }^{38}$ Similarly, a randomized controlled trial was done to determine whether algorithm-driven telephone care by nurses improved lipid control in patients with diabetes. During this 20 -month study, nurses contacted patients by phone and used algorithms to assess lipid values and initiate and titrate lipid-lowering medications. Patients in the experimental group improved lipid control and decreased overall health care utilization..$^{45}$

To assess the effect of web-based care management on glucose and blood pressure control in patients with poorly controlled diabetes, participants attended a half-day diabetes self-management education class and were then randomized to the usual care or intervention group. The intervention group also received a notebook computer, glucose meter, and blood pressure monitor, and could message the care manager, upload glucose and blood pressure information, and view educational modules. Participants in the intervention group had significant decreases in $\mathrm{HbA}_{1 \mathrm{c}}$ and systolic blood pressure, and a significant difference was found between the intervention and control groups. ${ }^{28}$ Another study that utilized the web to deliver diabetes self-management education as an alternative to group classes found significant improvements in $\mathrm{HbA}_{1 \mathrm{c}}$ compared with the control group who attended traditional diabetes self-management education classes. ${ }^{17}$

While many studies have found significant improvements in $\mathrm{HbA}_{1 \mathrm{c}}$ following nursing intervention, other studies have noted no change or no difference between experimental and control groups. . $^{12,19,20,27,46}$ Further, some studies have found improvement in other physiologic outcomes for both intervention and control groups. ${ }^{6,13,44}$

\section{Psychosocial outcomes}

Psychosocial screening, including attitudes about diabetes, depression, and diabetes-related anxiety and assessment of quality of life, should be ongoing for people living with diabetes. Emotional well-being is associated with positive diabetes outcomes. ${ }^{1}$ Nursing intervention studies address psychosocial factors and evaluate the effect of interventions on these factors.

One study used a nurse-conducted motivational interviewing intervention to encourage participants to discuss self-management behaviors and explore their feelings about change. Both the intervention and control groups received multidisciplinary education and attended a diabetes club that facilitated group discussion of experiences of living with diabetes. Following the intervention, significant improvement was noted in quality of life for the experimental group, but not the control group. Both groups had significantly decreased mean scores for depression, anxiety, and stress, with no significant difference between groups. The lack of difference between the two groups may be attributed to exposure of the control group to education and the diabetes club support group. ${ }^{47}$ In a nurse-directed intervention to promote healthy behaviors in adults with diabetes, mean scores for psychologic-spiritual, social, and family domains in the experimental group were significantly higher when compared with the control group. ${ }^{3}$ A study using a tailored educational and goal-setting program guided by a nurse noted significant improvements in quality of life. ${ }^{29}$ Following a study in which participants received diabetes symptom-focused management intervention modules delivered by a nurse, perceptions of quality of life significantly increased and distress from symptoms significantly decreased. ${ }^{16}$

\section{Discussion}

The literature indicates that nurses are involved in a variety of interventions aimed at improving diabetes outcomes. Nurses are participating in diabetes research as primary investigators and members of the research team. They are responsible for delivering interventions, supervising delivery of care by other health care professionals, and coaching community health advisors. A key nursing intervention is provision of education for individuals living with diabetes. Educational interventions are delivered using various methods, including face-to-face individual or group sessions, telephone sessions, and web-based sessions. Benefits of nursing interventions for individuals living with diabetes include increased diabetes knowledge, increased participation in self-management behaviors, improved glycemic control, and improved psychosocial outcomes.

The current review uses an integrative approach rather than a systematic analysis. Conclusions based on the review may be limited based on the integrative approach used. The majority of studies included in this review were randomized controlled trials; however, some studies included a small sample size that may affect the generalizability of study findings. ${ }^{8,14,18,20,23,29}$ Additional limitations to generalizability exist due to the wide variety of geographic locations and health care environments in which studies were conducted.

A chief concern with many studies is the absence of clearly described nursing roles and activities. Repetition of 
nursing intervention studies to provide further evidence of effectiveness is difficult or impossible without a comprehensive description of the nurse's role and activities. Recommendations for improvements, interventions, and activities of nurses and other interventionists should be well described to assist with decisions about future nursing interventions for diabetes care.

Improvements are noted in overall glycemic control in some nursing intervention studies, but not all. This could be related to the short duration of some studies, that may not allow for adequate time to note long-term behavior changes. Further research is needed with longer follow-up periods to establish knowledge about the effect of nursing interventions over time. In addition, it is necessary to determine whether improvements in diabetes outcomes can be maintained after the intervention period or if ongoing intervention is needed to maintain diabetes self-management behaviors.

Some studies note improvements in outcomes for both intervention and control groups. This could be due to awareness through study involvement of the need to improve diabetes management. Another possible reason for improvement in both groups could be the delivery of an alternate intervention, such as educational materials, for the control group. ${ }^{6,37}$

\section{Conclusion}

Diabetes self-management activities are essential for individuals living with diabetes. Management of the complex diabetes treatment regimen requires knowledge, motivation, training, and support, which can be provided by nurses. ${ }^{37}$ The majority of nursing interventions included in this review contain educational and support components designed to equip people living with diabetes with the tools necessary to manage their chronic disease. Nurses work with patients to establish self-management goals and implement strategies to support positive self-management behaviors in the areas of physical activity, healthy eating, medicationtaking, self-monitoring of glucose, active participation in screening for complications, and effective coping. Nursing interventions improve patient outcomes, including diabetes knowledge, self-management behaviors, glycemic control, and quality of life. The findings from this review support the need for continued nursing intervention in diabetes care and suggest that further research is needed to establish the most effective, evidence-based interventions.

\section{Disclosure}

The author reports no conflict of interest in this work.

\section{References}

1. American Diabetes Association. Standards of medical care in diabetes - 2013. Diabetes Care. 2013;36 Suppl 1:s11-s66.

2. Carey N, Courtenay M. A review of the activity and effects of nurse-led care in diabetes. J Nurs Healthc Chronic Illn. 2007;16:296-304.

3. DeSouza MS, Nairy KS. An interventional study on the health promoting behaviours of adults with diabetes. Clin Eff Nurs. 2004;8:68-80.

4. Bodenheimer T, MacGregor K, Stothart N. Nurses as leaders in chronic care. BMJ. 2005;330:612-613.

5. Guyse LA, McHugh BR, Meszaros JF, et al. Collaborative approach to implementing and adapted diabetes prevention program lifestyle intervention. Diabetes Spectr. 2011;24:138-147.

6. Sherifall D, Greb JL, Amirthavasar G, et al. Effect of computer-generated tailored feedback on glycemic control in people with diabetes in the community. Diabetes Care. 2011;34:1794-1798.

7. Shi Q, Ostwald SK, Wang S. Improving glycaemic control self-efficacy and glycaemic control behaviour in Chinese patients with type 2 diabetes mellitus: randomised controlled trial. J Clin Nurs. 2010;19:398-404.

8. Kang CM, Chang SC, Chen PL, et al. Comparison of family partnership intervention care vs conventional care in adult patients with poorly controlled type 2 diabetes in a community hospital: a randomized controlled trial. Int J Nurs Stud. 2010;47:1363-1373.

9. Wu SF, Lee MC, Liang SY, Lu YY, Wang TJ, Tung HH. Effectiveness of a self-efficacy program for persons with diabetes: a randomized controlled trial. Nurs Health Sci. 2011;13:335-343.

10. Brown SA, Garcia AA, Kouzekanani K, Hanis CL. Culturally competent diabetes self-management education for Mexican Americans. Diabetes Care. 2002;25:259-268.

11. Gary TL, Bone LR, Hill MN, et al. Randomized controlled trial of the effects of nurse case manager and community health worker interventions on risk factors for diabetes-related complications in urban African Americans. Prev Med. 2003;37:23-32.

12. McEwen MM, Pasvogel A, Gallegos G, Barrera L. Type 2 diabetes self-management social support intervention at the US-Mexico border. Public Health Nurs. 2010;27:310-319.

13. Skelly AH, Carlson JR, Leeman J, Soward A, Burns D. Controlled trial of nursing interventions to improve health outcomes of older African American women with type 2 diabetes. Nurs Res. 2009;58:410-418.

14. Vincent D. Culturally tailored education to promote lifestyle change in Mexican Americans with type 2 diabetes. J Am Acad Nurse Pract. 2009;21:520-527.

15. New N. Teaching so they can hear: using a co-created diabetes selfmanagement education approach. J Am Acad Nurse Pract. 2010;22: 316-325.

16. Skelly AH, Carlson JR, Leeman J, Holditch-Davis D, Soward ACM. Symptom-focused management for African American women with type 2 diabetes: a pilot study. Appl Nurs Res. 2005;18:213-220.

17. Song M, Choe MA, Kim KS, et al. An evaluation of web-based education as an alternative to group lectures for diabetes self-management. Nurs Health Sci. 2009;11:277-284.

18. Sigurdardottir AK, Benediktsson R, Jonsdottir H. Instruments to tailor care of people with type 2 diabetes. J Adv Nurs. 2009;65:2118-2130.

19. Walker EA, Stevens KA, Persaud S. Promoting diabetes self-management among African Americans: an educational intervention. J Health Care Poor Underserved. 2010;21:169-186.

20. Huang JP, Chen HH, Yeh ML. A comparison of diabetes learning with and without interactive multimedia to improve knowledge, control, and self-care among people with diabetes in Taiwan. Public Health Nurs. 2009;26:317-328.

21. Walker EA, Shmukler C, Ullman R, Blanco E, Scollan-Koliopoulus M, Cohen HW. Results of a successful telephonic intervention to improve diabetes control in urban adults. Diabetes Care. 2011;34:2-7.

22. Davis RM, Herman WH, Hitch AD, Mayer-Davis EJ, Salaam MM, Zimmer-Galler IE. Telehealth improves diabetes self-management in an underserved community: diabetes telecare. Diabetes Care. 2010;33:1712-1717. 
23. Kim HS, Oh JA. Adherence to diabetes control recommendations: impact of nurse telephone calls. J Adv Nurs. 2003;44:256-261.

24. Tang TS, Funnell MM, Noorulla S, Oh M, Brown MB. Sustaining short-term improvements over the long-term: results from a 2-year diabetes self-management support (DSMS) intervention. Diabetes Res Clin Pract. 2012;95:85-92.

25. Evans MM. Evidence-based practice protocol to improve glucose control in individuals with type 2 diabetes mellitus. MedSurg Nurs. 2010;19:317-322.

26. Gallegos EC, Berumen FO, Gomez-Meza MV. Metabolic control of adults with type 2 diabetes mellitus through education and counseling. J Nurs Scholarsh. 2006;38:344-351.

27. Rygg LO, Rise MB, Gronning K, Steinsbekk A. Efficacy of ongoing group based diabetes self-management education for patients with type 2 diabetes mellitus. A randomized controlled trial. Patient Educ Couns. 2012;86:98-105.

28. McMahon GT, Gomes HE, Hohne SH, Hu TM, Levine BA, Conlin PR. Web-based care management in patients with poorly controlled diabetes. Diabetes Care. 2005;28:1624-1629.

29. Moriyama M, Nakano M, Kuroe Y, Nin K, Niitani M, Nakaya T. Efficacy of a self-management education program for people with type 2 diabetes: results of a 12 month trial. Jpn J Nurs Sci. 2009;6:51-63.

30. Handley MA, Shumway M, Schillinger D. Cost-effectiveness of automated telephone self-management support with nurse care management among patients with diabetes. Ann Fam Med. 2008;6:512-518.

31. Tan MY, Magarey JM, Chee SS, Lee LF, Tan MH. A brief structured education programme enhances self-care practices and improves glycaemic control in Malaysians with poorly controlled diabetes. Health Educ Res. 2011;26:896-907.

32. Wu SF, Liang SY, Wang TJ, Chen MH, Jian YM, Cheng KC. A selfmanagement intervention to improve quality of life and psychosocial impact for people with type 2 diabetes. J Clin Nurs. 2011;20: 2655-2665.

33. Schillinger D, Handley M, Wang F, Hammer H. Effects of self-management support on structure, process, and outcomes among vulnerable patients with diabetes. Diabetes Care. 2009;32:559-566.

34. Turner J, Larsen M, Tarassenko L, Neil A, Farmer A. Implementation of telehealth support for patients with type 2 diabetes using insulin treatment: an exploratory study. Inform Prim Care. 2009;17:47-53.

35. Radhakrishnan $\mathrm{K}$. The efficacy of tailored interventions for self-management outcomes of type 2 diabetes, hypertension, or heart disease: a systematic review. $J A d v$ Nurs. 2012;68:496-510.
36. Thoolen BJ, de Ridder D, Bensing J, et al. Effectiveness of a selfmanagement intervention in patients with screen-detected type 2 diabetes. Diabetes Care. 2007;30:2832-2837.

37. Frosch DL, Uy V, Ochoa S, Mangione CM. Evaluation of a behavior support intervention for patients with poorly controlled diabetes. Arch Intern Med. 2011;171:2011-2017.

38. Thoolen BJ, de Ridder D, Bensing J, Gorter K, Rutten G. Beyond good intentions: the role of proactive coping in achieving sustained behavioural change in the context of diabetes management. Psychol Health. 2009;24:237-254.

39. Albright A, Araujo R, Brownson C, et al. AADE position statement: community health workers in diabetes management and prevention. 2009. Available from: https://www.aadenet.org/export/sites/aade/_ resources/pdf/CommunityHealthWorkerPositionStatement2009.pdf. Accessed July 10, 2013.

40. Young RJ, Taylor J, Friede T, et al. Pro-Active Call Center Treatment Support (PACCTS) to improve glucose control in type 2 diabetes. Diabetes Care. 2005;28:278-282.

41. Wu CJ, Chang AM, Courtney M, Ramis MA. Using user-friendly telecommunications to improve cardiac and diabetes self-management programme: a pilot study. J Eval Clin Pract. 2012;18:695-697.

42. Sturt J, Whitlock S, Hearnshaw H. Complex intervention development for diabetes self-management. JAdv Nurs. 2006;54:293-303.

43. Funnell MM, Weiss MA. Empowering patients with diabetes Nursing. 2009;39:34-37.

44. Weinger K, Beverly EA, Lee Y, Sitnokov L, Ganda OP, Caballero E. The effect of a structured behavioral intervention on poorly controlled diabetes. Arch Int Med. 2011;171:1990-1999.

45. Fischer HH, Eisert SL, Everhart RM, et al. Nurse-run, telephone-based outreach to improve lipids in people with diabetes. Am J Manag Care. 2012;18:77-84.

46. Gabbay RA, Lendel I, Saleem TM, et al. Nurse case management improves blood pressure, emotional distress and diabetes complication screening. Diabetes Res Clin Pract. 2006;71:28-35.

47. Chen SM, Creedy D, Lin HS, Wollin J. Effects of motivational interviewing intervention on self-management, psychological and glycemic outcomes in type 2 diabetes: a randomized controlled trial. Int $J$ Nurs Stud. 2012;49:637-644.
Nursing: Research and Reviews

\section{Publish your work in this journal}

Nursing: Research and Reviews is an international, peer-reviewed, open access journal publishing original research, reports, reviews and commentaries on all aspects of nursing and patient care. These include patient education and counselling, ethics, management and organizational issues, diagnostics and prescribing, economics and

\section{Dovepress}

resource management, health outcomes, and improving patient safety in all settings. The manuscript management system is completely online and includes a very quick and fair peer-review system. Visit http://www.dovepress.com/testimonials.php to read real quotes from published authors. 